Journal of Systems Science and Information

Jun., 2016, Vol. 4, No. 3, pp. 195-211

DOI: $10.21078 / J S S I-2016-195-17$

\title{
Capability Oriented Combat System of Systems Networked Modeling and Analyzing
}

\author{
Qingsong ZHAO* \\ School of Information System and Management, National University of Defense Technology, \\ Changsha 410073, China \\ E-mail: zhaoqingsong@nudt.edu.cn
}

Xiaoke ZHANG

School of Information System and Management, National University of Defense Technology, Changsha 410073, China; Leiden Institute of Advanced Computer Science, Leiden University, Leiden 2333CA, Netherlands

E-mail: zhangxiaoke2013@hotmail.com

\section{Zhiwei YANG}

School of Information System and Management, National University of Defense Technology, Changsha 410073, China; Leiden Institute of Advanced Computer Science, Leiden University, Leiden 2333 CA, Netherlands

E-mail: zyang@liacs.nl

\begin{abstract}
Combat modeling is an important area of military operations. System of system counterwork is an important mode of information-based war which is a mode of "network centered" instead of "platform centered" and "capability oriented" instead of "function oriented". Under the conditions of informationization, the combat model must therefore address these challenges by properly representing the networked efficient based on mutual relations among combat entities. The implementation process of combat system of systems capability is analyzed which is the result of complex interactions between the entities in four domains through a sequence of action processes. The combat network model of combat system of systems is described which reflects the fundamental structure of combat system of systems. The entity with three types of functions and five types of relations in the combat network is analyzed. The capability loop is defined and the evaluation index of combat network of combat system of systems is proposed based on the capability loop analysis. Finally, an example is used to illustrate the methodology.
\end{abstract}

Keywords combat system of systems; network modeling; capability loop; evaluation and analysis

\section{Introduction}

Under the conditions of informationization, the combat mode is transformed from the industrial age combat to the information age combat, from "platform centered" to "network centered",

Received September 1, 2015, accepted October 30, 2015

*Corresponding author

Supported by National Natural Science Foundation of China (71331008, 71571185) and National Social Science Foundation of China in Military Science (15GJ003-278) 
and the operation takes the behaviors of counterwork between system of systems (SoS). The results of the combat depend on the combat capability of all weapons and equipment, and the overall combat capability depends on the interaction of multiple systems rather than an individual attribute of the weapons and equipment ${ }^{[1-3]}$.

SoS is a collection of task-oriented or dedicated systems that pool their resources and capabilities together to create a new, more complex system which offers more functionality and better performance than simply the sum of the constituent systems ${ }^{[4,5]}$. Combat system of systems (CSoS) is a large, complex, enduring collection of interdependent weapon systems under development over time by multiple independent authorities to provide multiple, interdependent capabilities to support multiple missions ${ }^{[6-9]}$.

Industrial age combat was characterized by limited communications, massed forces, and centralized command, control and decision making. Since the information was difficult to obtain and hard to share, commanders relied more on forces than on a deep insight into tactical intricacies. Information technology and computer networks have been introduced into military processes to improve on this brute force approach by exchanging information for physical force where appropriate. As a result of these efforts, military systems are increasingly characterized by dispersal of physical assets, information distribution and decentralized cognition. A new value system is emerging where the arrangement of distributed, networked assets is more important than mere massing of force ${ }^{[10-12]}$.

Network-centric warfare (NCW) is the concept developed by the United States to describe the paradigm shift central to the revolution in US military affairs. It is defined as an information superiority-enabled concept of operations that generates increased combat power by networking sensors, decision makers, and shooters to achieve shared awareness, increased speed of command, higher tempo of operations, greater lethality, increased survivability and a degree of self- synchronization ${ }^{[1,13]}$. There are large amount of matter and energy interaction based on information interaction in each part of CSoS. The combat efficient of CSoS takes representation of networked efficient based on these mutual relationships. Therefore, combat efficient of CSoS under the conditions of informationization has to manifest the whole network emergent effect caused by the complex interactions among the entities ${ }^{[2,14]}$.

An information age CSoS model requires a transformation in military modeling philosophy and must therefore addresses these challenges by properly representing complex local behaviors, explicitly representing interdependencies and capturing the skewed distribution of networked performance. In addition, an information age CSoS model must capture both the attrition processes and the search and detection processes important to distributed, networked warfare. Such a model would be a bona fide transformation in combat modeling philosophy and constitute a true information age $\mathrm{CSoS}$ model ${ }^{[10]}$.

The objective of this paper is to propose an information age CSoS model that satisfies the requirements of the transformation in combat modeling. The rest of the paper is organized as follows. A literature review of related work is presented in Section 2. Section 3 describes the implementation of CSoS capability. Section 4 is dedicated to the CSoS network model. Section 5 demonstrates the evaluation of CSoS network model. Section 6 shows the example of CSoS network model and evaluates the results. And finally, the work is concluded in Section 7. 


\section{Related Work}

Although there is a great variety in the specific application of combat models ${ }^{[15,16]}$, the fundamental structure of most combat models is one of the two basic types: deterministic (closed-form) or stochastic (probability-based) combat models [10]. The most prevalent example of a deterministic combat model is the eponymous Lanchester equations ${ }^{[17-19]}$. This model is the basis for most of the current attrition-based combat simulations in use today. Indeed, there are dozens of variants of Lanchesters model representing ground or air combat processes in use today ${ }^{[20-22]}$. A model that explicitly includes randomness and uncertainty is called a stochastic mode ${ }^{[23]}$. Stochastic models are usually created by modifying one or more of the terms in a deterministic equation with random draws from some probability distribution. Traditional stochastic combat models represent combat as a chain of independent events (each with their own probability of occurrence) or as sets of basic interaction equations (with random variables representing operational processes) $)^{[24,25]}$.

Those models cannot take account of relationships among the inner units of the combat system, and lack description of apperception, information interaction, command and control, cooperation in the information battlefield ${ }^{[14]}$. Thus, a description framework can reflect the incorporate and networked oppositional operation of the combat SoS. It is necessary to find out a new description method of combat efficient of SoS.

Some defense analysts continue to use traditional models to simulate new, information age operational concepts ${ }^{[26-28]}$. Sometimes these models are embellished with additional C2 parameters (in the case of deterministic models) or the addition of $\mathrm{C} 2$ statistical terms (in the case of stochastic models) ${ }^{[29,30]}$. However, the underlying philosophy of these models has not departed from merely modifying traditional attrition models with $\mathrm{C} 2$ parameters or processes. Existing models have failed to represent the impact of new forms of command and control on combat outcomes because they are all based on physical models of attrition ${ }^{[10]}$. For this reason, none of the common models discussed here are suitable candidates for an information age combat model.

A newer set of models has been described by researchers attempting to add more specificity to the concept of network centric warfare, the predominant theory of warfare in the information age ${ }^{[31-33]}$. Modern warfare has the exhibition of counterwork between SoS based on the more and more extrusive network. So it is rational to describe the oppositional operation of the combat from the point of network science view. Network analysis has emerged as a powerful way of studying phenomena as diverse as interpersonal interaction, connections among neurons, the structure of the internet ${ }^{[34,35]}$, and military networks ${ }^{[36,37]}$. Complex network theory is a crossed subject which combines wholeness, complexity and network organically and mines the essential characteristics of complex system through simulation, dynamics evolution and statistical physics ${ }^{[38]}$. It becomes a new theoretic method to explore complexity in the networkcentric combat ${ }^{[39,40]}$. The power network-centric warfare is the results of the increase in speed of command, self-synchronization of forces, and higher situational awareness. OODA loop is an information strategy concept from information warfare. In OODA loop, military operations are basically a sequence of decision processes or cycles. These cycles have four main points: Observe, orient, decide, and act. OODA loop shows that all decisions are based on observations 
of the evolving situation. These observations are the raw information on which decisions and actions are based. The observed information must be processed to be oriented for making a further decision ${ }^{[41,42]}$.

An information age combat model requires a transformation in military modeling philosophy and must therefore addresses these challenges by properly representing complex local behaviors, explicitly representing interdependencies and capturing the skewed distribution of networked performance. In addition, an information age combat model must capture both the attrition processes and the search and detection processes important to distributed, networked warfare.

\section{CSoS Capability Implementation}

CSoS is the large, complex, enduring collections of interdependent systems under development over time by multiple independent authorities to provide multiple, interdependent capabilities to support multiple missions. The behavior of CSoS depends not only on the constituent systems but also on the complex interactions between the constituent systems. The number of connections between constituent systems, the diversity of the constituent systems and the way of the constituent systems organized can lead to different emergent CSoS behaviors.

Capability is the ability to execute a specified course of action ${ }^{[43]}$. CSoS emphasizes the capability of CSoS rather than the function of the individual system in CSoS. CSoS capability is the ability of CSoS to achieve an effect under specified conditions through multiple combinations of means and ways to perform a set of tasks such as delivering fire on a target or delivering electronic interference on a target.

The capability implementation of CSoS is the result of a variety of combat elements in certain combat process interaction including the physical element such as the firepower, the information element such as the intelligence, the cognitive element such as the commander, and the organization element such as the cooperation. The capability implementation of CSoS is the result of complex interactions between the entities in four domains including the physical domain (PD), information domain (ID), cognitive domain (CD) and organization domain (OD). PD is composed of the entities with the function of attack or interference aiming at delivering fire or electronic interference on the target. ID is composed of the entities with the function of reconnaissance, surveillance and early warning aiming to at getting and delivering targets information. CD is composed of the entities with the function of command and control. OD is composed of the relations among the entities in PD, ID and CD concerned about the collaboration between the entities with various functions.

The capability implementation of CSoS is basically a sequence of action processes. These processes have three main actions: observation, decision and action. Observation part is a stage where surrounding information about the tasks is observed. In the decision part, deciders make decisions about the present and future arrangement of the other parts based on the information from the observation part. In the action part, the decision is executed such as delivering fire on the target by the missile or delivering electronic interference on the target by the electronic jammer.

The capability implementation of CSoS can be shown in Figure 1. At the macroscopic level, the capability implementation of CSoS is the result of the cooperation between the PD, ID, 
$\mathrm{CD}$ and OD. At the microcosmic level, the capability implementation of CSoS is the result of a combat processes including the observation, decision and action.

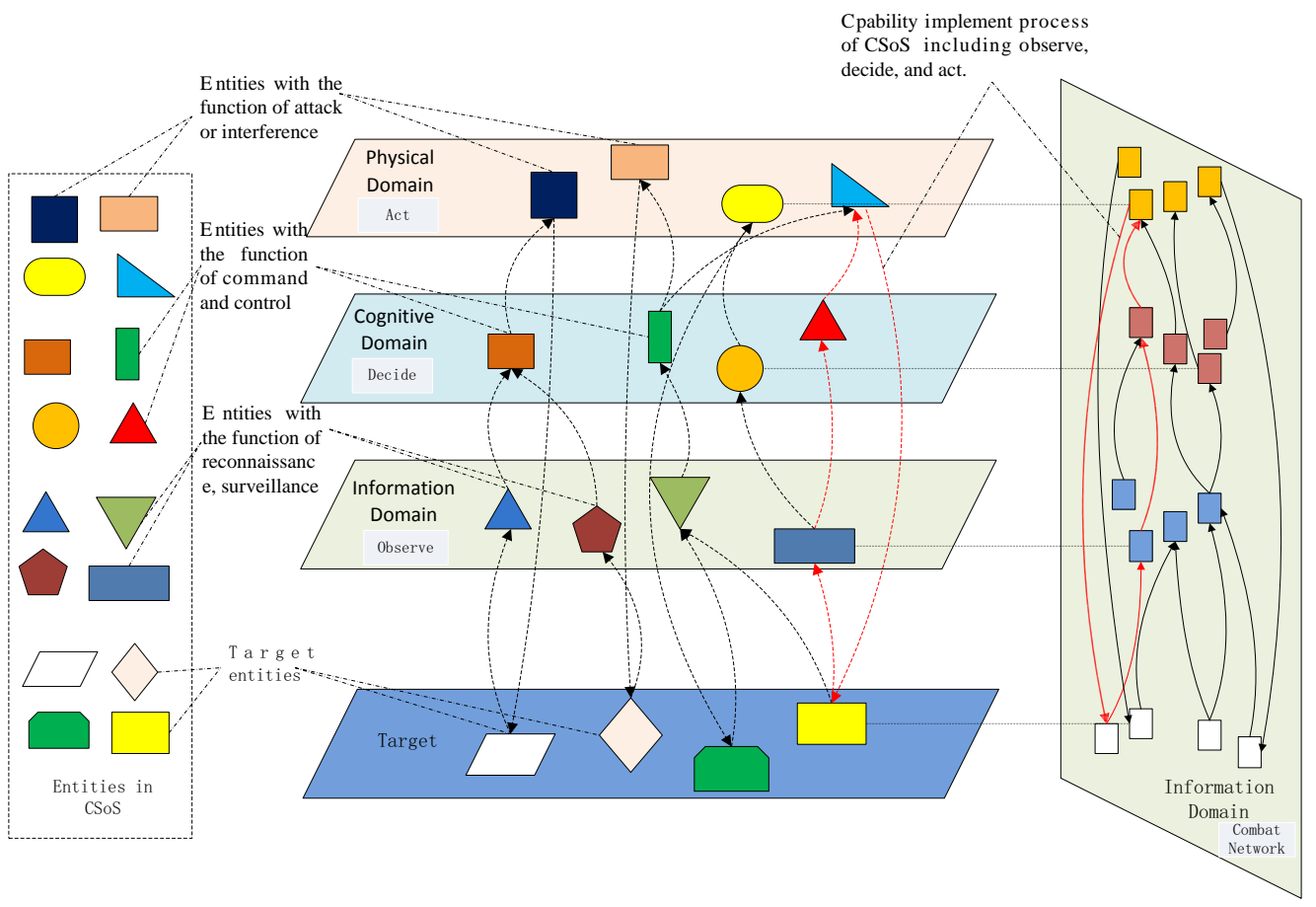

Figure 1 The capability implementation of CSoS

\section{Combat Network Model of CSoS}

The CSoS can be represented as a combat network described by OD. The node is the target entity and the entities in PD, ID and CD. The link is the relationships between the entities. Combat network reflects the incorporate and networked oppositional operation of CSoS.

The combat network model of CSoS can be described as $G=\left(V^{\prime}, T, E^{\prime}, F, F^{\prime}\right)$.

1) $V^{\prime}$ is the collection of entities in PD, ID and CD;

2) $T$ is the collection of target entities, $V=V^{\prime} \cup T, N$ is the number of entities in $V$;

3) $E$ is the collection of relations between the entities in $V$, a relation from $v_{i}$ to $v_{j}, v_{i}, v_{j} \in V$ is denoted by $e_{i j}$ and $e_{i j} \in E$;

4) $F$ is the collection of the entity attributes, $F=\left\{F_{1}, F_{2}, \cdots, F_{i}, F_{N}\right\} . F_{i}=\left[f\left(v_{i}\right), f_{1}\left(v_{i}\right)\right.$, $\left.f_{2}\left(v_{i}\right), f_{3}\left(v_{i}\right), f_{4}\left(v_{i}\right)\right]$ is the collection of the attribute of $v_{i}, v_{i} \in V . f\left(v_{i}\right)$ is the function type of $v_{i}$. If $v_{i} \in V^{\prime}$, then $f\left(v_{i}\right) \subseteq$ \{Sensor, Decsion, Influence . $f_{1}\left(v_{i}\right)$ is the function of $v_{i}, f_{2}\left(v_{i}\right)$ is the reaction time of $v_{i}, f_{3}\left(v_{i}\right)$ is the reliability of $v_{i}, f_{4}\left(v_{i}\right)$ is the cost of $v_{i}$. Let $f_{i}(v) \in[0,1]$, $i=1,2,3,4, v \in V$. The stronger of the function is, the more $f_{1}(v)$ closes to 1 , the greater of the entity supports the capability. The shorter of the reaction time is, the more $f_{2}(v)$ close to 0 , the greater of the entity supports the capability. The higher of the reliability is, the more $f_{3}(v)$ closes to 1 , the greater of the entity supports the capability. The more of the cost, the more $f_{4}(v)$ close to 1 . If $v_{i} \in T$, then $f\left(v_{i}\right)$ is the value of $v_{i}$ and $f_{i}\left(v_{i}\right)=\emptyset, i=1,2,3$; 
5) $F^{\prime}$ is the collection of the relation attributes, $F^{\prime}=\left\{F_{i j}\right\} . F_{i j}=\left[f^{\prime}\left(e_{i j}\right), f_{1}^{\prime}\left(e_{i j}\right), f_{2}^{\prime}\left(e_{i j}\right)\right.$, $\left.f_{3}^{\prime}\left(e_{i j}\right), f_{4}^{\prime}\left(e_{i j}\right)\right]$ is the collection of the attributes of $e_{i j} \in E$. $f^{\prime}\left(e_{i j}\right)$ is the function type of $e_{i j}$. $f_{1}^{\prime}\left(e_{i j}\right)$ is the function of $e_{i j}$ and $f^{\prime}\left(e_{i j}\right) \in\left\{R_{T-S}, R_{S-D}, R_{D-I}, R_{I-T}, R_{S-S}, R_{D-D}, R_{I-I}\right\}$. $f_{2}^{\prime}\left(e_{i j}\right)$ is the reaction time of $e_{i j}, f_{3}^{\prime}\left(e_{i j}\right)$ is the reliability of $e_{i j}, f_{4}^{\prime}\left(e_{i j}\right)$ is the cost of $e_{i j}$. Let $f_{i}^{\prime} e_{i j} \in[0,1], i=1,2,3,4, e_{i j} \in E$. The stronger of the function is, the more $f_{1}^{\prime}\left(e_{i j}\right)$ close to 1 , the greater of the relation supports the capability. The shorter of the reaction time is, the more $f_{2}^{\prime}\left(e_{i j}\right)$ close to 0 , the greater of the relation supports the capability. The higher of the reliability is, the more $f_{3}^{\prime}\left(e_{i j}\right)$ close to 1 , the greater of the relation supporting supports the capability is. The more of the cost is, the more $f_{4}^{\prime}\left(e_{i j}\right)$ close to 1 , the more of the cost supports the capability.

The function of the entity in PD, ID and DC can be divided into three types: Sensor function, decision function and influence function.

1) Senor function is the function of reconnaissance, surveillance and early warning etc. The entity with the sensor function is the sensor entity. The detection range and quality of the sensor entity decide the support degree of the sensor entity for the CSoS capability implementation.

2) Decision function is the function of decision based on the information observed by the sensor entity. The entity with the decision function is the decision entity. The degree of the information processing and situational awareness of the decision entity decide the support degree of the decision entity for the CSoS capability implementation.

3) Influence function is the function of fire attack or electronic interference based on the information observed by the sensor entity and the decision by the decision entity. The entity with the influence function is the influence entity. The degree of the fire attack or electronic interference of the influence entity decides the support degree of the influence entity for the CSoS capability implementation.

Let $S=\left\{x \mid x \in V^{\prime} \wedge\right.$ Sensor $\left.\in f(x)\right\}, D=\left\{x \mid x \in V^{\prime} \wedge\right.$ Decsion $\left.\in f(x)\right\}, I=\{x \mid x \in$ $V^{\prime} \wedge$ Influence $\left.\in f(x)\right\}$ is the collection of entities with the function of sensor, decision and influence.

The relationship between the entities in PD, ID and DC can be divided into five types: Sensor relation, information relation, command relation, influence relation and cooperation relation.

1) Sensor relation is the relation between the sensor entity and the target, denoted by $R_{T-S}$. The direction of sensor relation is from the target to the sensor entity.

2) Information relation is the relation between the decision entity and sensor entity, denoted by $R_{S-D}$. The direction of information relation is from the sensor entity to the decision entity.

3) Command relation is the relation between the influence entity and the decision entity, denoted by $R_{D-I}$. The direction of command relation is from the decision entity to the influence entity.

4) Influence relation is the relation between the influence entity and the target, denoted by $R_{I-T}$. The direction of sensor relation is from the influence entity to the target.

5) Cooperation relation is the relation between the entities with the same function type. $R_{S-S}$ is the relation between the sensor entities. $R_{D-D}$ is the relation between the decision entities. $R_{I-I}$ is the relation between the influence entities. For example, when two sensor en- 
tities conduct reconnaissance on the same target, the reconnaissance accuracy can be improved if two sensor entities can share the reconnaissance information. The direction of cooperation relation is from the entity which starts the cooperation firstly.

$$
= \begin{cases}-\ln \left[k_{i j} \frac{f_{1}\left(v_{j}\right) f_{3}\left(v_{j}\right)}{f_{2}\left(v_{j}\right)}+k_{i j}^{\prime} \frac{f_{1}^{\prime}\left(e_{i} j\right) f_{3}^{\prime}\left(e_{i} j\right)}{f_{2}^{\prime}\left(e_{i} j\right)}\right], & f^{\prime}\left(e_{i j}\right)=R_{T-S}, \\ -\ln \left[k_{i j} \frac{f_{1}\left(v_{i}\right) f_{3}\left(v_{i}\right)}{f_{2}\left(v_{i}\right)}+k_{i j}^{\prime} \frac{f_{1}^{\prime}\left(e_{i} j\right) f_{3}^{\prime}\left(e_{i} j\right)}{f_{2}^{\prime}\left(e_{i} j\right)}\right], & f^{\prime}\left(e_{i j}\right)=R_{I-T}, \\ -\ln \left[k_{i j}^{i} \frac{f_{1}\left(v_{i}\right) f_{3}\left(v_{i}\right)}{f_{2}\left(v_{i}\right)}+k_{i j}^{j} \frac{f_{1}\left(v_{j}\right) f_{3}\left(v_{j}\right)}{f_{2}\left(v_{j}\right)}+k_{i j}^{\prime} \frac{f_{1}^{\prime}\left(e_{i} j\right) f_{3}^{\prime}\left(e_{i} j\right)}{f_{2}^{\prime}\left(e_{i} j\right)}\right], & f^{\prime}\left(e_{i j}\right) \in U, \\ \text { where } U=\left\{R_{S-D}, R_{D-I}, R_{S-S}, R_{D-D}, R_{I-I}\right\} . & \end{cases}
$$

For $e_{i j} \in E$, let $W\left(e_{i j}\right)=\left[w_{1}\left(e_{i j}\right), w_{2}\left(e_{i j}\right)\right], w_{1}\left(e_{i j}\right) \in(0,1), w_{1}\left(e_{i j}\right) \in(0,1) . w_{1}\left(e_{i j}\right)$ is the implementation degree of the operational activity $e_{i j} . w_{2}\left(e_{i j}\right)$ is the cost of the implementation of the operational activity $e_{i j} . k_{i j}^{i} \in(0,1), k_{i j}^{j} \in(0,1)$ is the weight of the entities and the relations in the operational activity $e_{i j}$ for the implementation of the capability.

The CSoS capability is supported by the cooperation between the entities in the PD, ID, CD and OD in the combat network. The capability depends not only on the entities but also on the cooperative mode between the entities. The implement degree of the operational activity $e_{i j}$ is defined as shown in equation (1). The cooperation can enhance individual entity capability and the CSoS capability is not the simple sum of the single capability proposed by the entities.

If $f^{\prime}\left(e_{i j}\right)=R_{T-S}, w_{1}\left(e_{i j}\right)$ is the uncertainty degree of the target information obtained by the sensor entity. The more of the information obtained by the sensor entity, the lower of the uncertainty is, the closer $w_{1}\left(e_{i j}\right)$ to 0 . If $f^{\prime}\left(e_{i j}\right)=R_{S-D}, w_{1}\left(e_{i j}\right)$ is the uncertainty degree of the target information obtained by the decision entity. The more of the information obtained by the decision entity, the lower of the uncertainty is, the closer $w_{1}\left(e_{i j}\right)$ to 0 is. If $f^{\prime}\left(e_{i j}\right)=R_{D-I}, w_{1}\left(e_{i j}\right)$ is the uncertainty degree of the decision made by the decision entity. The higher of the decision correctness is, the lower of the uncertainty is, the closer $w_{1}\left(e_{i j}\right)$ to 0 is. If $f^{\prime}\left(e_{i j}\right)=R_{I-T}, w_{1}\left(e_{i j}\right)$ is the uncertainty degree of destroying the target by the influence entity. The more destroying the target, the lower of the uncertainty is, the closer $w_{1}\left(e_{i j}\right)$ to 0 is. If $f^{\prime}\left(e_{i j}\right)=R_{S-S}, w_{1}\left(e_{i j}\right)$ is the uncertainty degree of the target information obtained cooperatively by two sensor entities. The higher of cooperation degree between two sensor entities is, the closer $w_{1}\left(e_{i j}\right)$ to 0 is. If $f^{\prime}\left(e_{i j}\right)=R_{D-D}, w_{1}\left(e_{i j}\right)$ is the uncertainty degree of the decision made cooperatively by two decision entities. The higher of the cooperation degree between two decision entities is, the closer $w_{1}\left(e_{i j}\right)$ to 0 is. If $f^{\prime}\left(e_{i j}\right)=R_{I-I}, w_{1}\left(e_{i j}\right)$ is the uncertainty degree of destroying the target cooperatively by two influence entities. The higher of the cooperation degree between two influence entities, the closer $w_{1}\left(e_{i j}\right)$ to 0 is.

The cooperation between the PD, ID, CD and OD, denoted by $e_{i j}$, will decrease the cost supporting the implementation of the capability. In general, the total cost will be smaller than any cost supporting the implementation of the capability by single entity. $w_{2}\left(e_{i j}\right)$ is defined as: 


$$
w_{2}\left(e_{i j}\right)= \begin{cases}\frac{f_{4}\left(v_{j}\right) \bullet f_{4}^{\prime}\left(e_{i} j\right)}{f_{4}\left(v_{j}\right)+f_{4}^{\prime}\left(e_{i} j\right)}, & f^{\prime}\left(e_{i j}\right)=R_{T-S}, \\ \frac{f_{4}\left(v_{j}\right) \bullet f_{4}^{\prime}\left(e_{i} j\right)}{f_{4}\left(v_{i}\right)+f_{4}^{\prime}\left(e_{i} j\right)}, & f^{\prime}\left(e_{i j}\right)=R_{I-T}, \\ \frac{f_{4}\left(v_{i}\right) \bullet f_{4}\left(v_{j}\right) \bullet f_{4}^{\prime}\left(e_{i} j\right)}{f_{4}\left(v_{i}\right)+f_{4}\left(v_{j}\right)+f_{4}^{\prime}\left(e_{i} j\right)}, & f^{\prime}\left(e_{i j}\right) \in\left\{R_{S-D}, R_{D-I}, R_{S-S}, R_{D-D}, R_{I-I}\right\}\end{cases}
$$
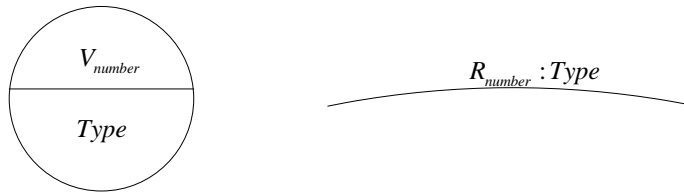

Figure 2 The entity and relation in the combat network of CSoS

The entity in the combat network of CSoS can be described as Figure 2(a). $V_{\text {number }}$ is the number of the entities and Type is the function type of the entity. If $v \in V^{\prime}$, then Type $\subseteq$ \{Sensor, Decsion, Influence\}. If $v \in T$, then Type=Target. The relation in the combat network of CSoS can be described as Figure 2(b). $R_{\text {number }}$ is the number of relations, Type is the type of the relation and Type $\in\left\{R_{T-S}, R_{S-D}, R_{D-I}, R_{I-T}, R_{S-S}, R_{D-D}, R_{I-I}\right\}$. Figure 2 (b) can be simplified to Figure 2(c).

The combat network of CSoS can be described as a directed network where $v \in V$ is the node and $e_{i j} \in E$ is the edge, shown in Figure 3. $P_{i j}=e_{i k_{1}}, e_{k_{1} k_{2}}, \cdots, e_{k_{m} k_{n}}, e_{k_{n} j}$ is a directed path in $G$ from node $v_{i}$ to node $v_{j}$. The length of the path is the number of edges in the path. $\bar{P}_{i j}$ is the collection of paths from node $v_{i}$ to node $v_{j} . V\left(P_{i j}\right)$ is the collection of the nodes in $P_{i j} . E\left(P_{i j}\right)$ is the collection of the edges in $P_{i j}$.

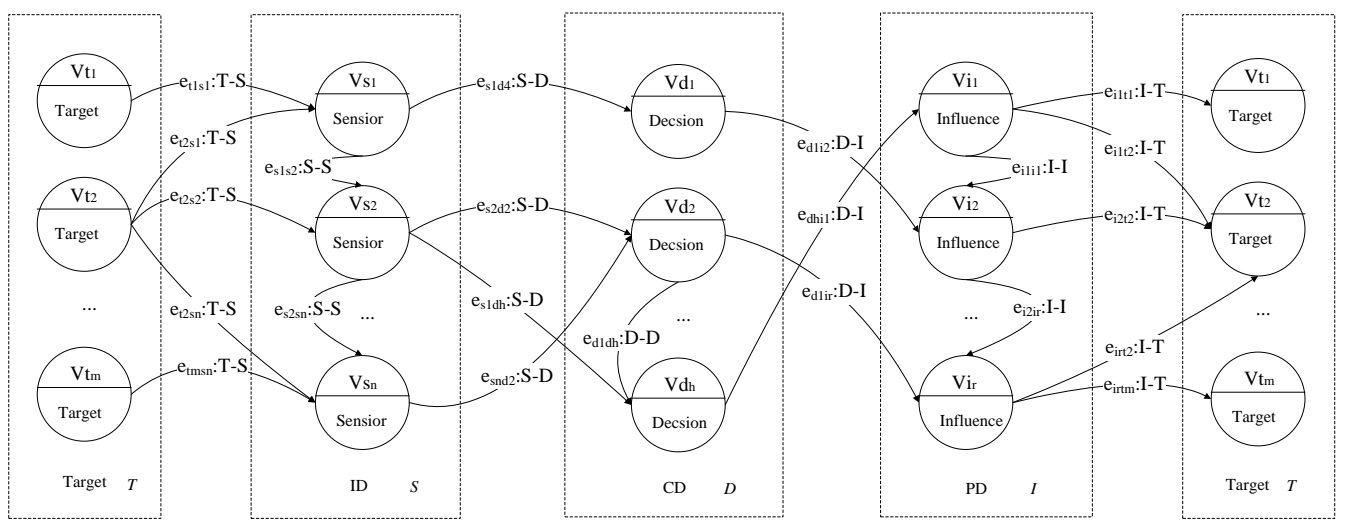

Figure 3 The combat network of CSoS

\section{Combat Network of CSoS Evaluation}

The implementation of the capability aim to attacking the target in CSoS depends not only on the cooperation among the entities in $\mathrm{PD}, \mathrm{CD}$ and ID but also on the process including 
observe, decide, and act. The implementation of the capability can be described as a path in the combat network of CSoS.

Definition 1 Capability loop for a target in the combat network is defined as a directed path $P_{t t}$ from the target to the target in the combat network of CSoS and denoted by $\Gamma$. Capability loop can be described as $P_{t t}=e_{t k_{1}} P_{k_{1} k_{2}} e_{k_{2} k_{3}} P_{k_{3} k_{4}} e_{k_{4} k_{5}} P_{k_{5} k_{6}} e_{k_{6} t}$ where $f\left(e_{t k_{1}}\right)=$ $R_{T-S}, f\left(e_{k_{2} k_{3}}\right)=R_{S-D}, f\left(e_{k_{4} k_{5}}\right)=R_{D-I}, f\left(e_{k_{6} T}\right)=R_{I-T}, v_{k_{1}} \in S, v_{k_{2}} \in S, v_{k_{3}} \in D$, $v_{k_{4}} \in D, v_{k_{5}} \in I, v_{k_{6}} \in I$. Obviously, $P_{k_{1} k_{2}}$ is the path in ID, $P_{k_{3} k_{4}}$ is the path in CD and $P_{k_{5} k_{6}}$ is the path in PD.

$\bar{\Gamma}\left(v_{t}\right)$ is the collection of capability loops for the target $v_{t} \in T . \Gamma_{i}\left(v_{t}\right)$ is the $i$-th capability loop in $\bar{\Gamma}\left(v_{t}\right) . V\left(\Gamma_{i}\left(v_{t}\right)\right)$ is the collection of entities in $\Gamma_{i}\left(v_{t}\right)$ and $E\left(\Gamma_{i}\left(v_{t}\right)\right)$ is the collection of relations in $\Gamma_{i}\left(v_{t}\right)$.

The evaluation index of $\Gamma_{i}\left(v_{t}\right)$ includes the uncertainty degree of the implementation of the capability and denote by $I\left(\Gamma_{i}\left(v_{t}\right)\right)$, the value of the implementation of the capability and denote by $Z\left(\Gamma_{i}\left(v_{t}\right)\right)$, the cost of the implementation of the capability and denote by $H\left(\Gamma_{i}\left(v_{t}\right)\right)$, the efficiency of the implementation of the capability and denote by $\Psi\left(\Gamma_{i}\left(v_{t}\right)\right)$.

The overall uncertainty degree of the implementation of the capability supported by $\Gamma_{i}\left(v_{t}\right)$ depends on the uncertainty degree of the various relationships in $\Gamma_{i}\left(v_{t}\right) . I\left(\Gamma_{i}\left(v_{t}\right)\right)$ is defined as:

$$
I\left(\Gamma_{i}\left(v_{t}\right)\right)=\sum_{e_{i j} \in E\left(\Gamma_{i}\left(v_{t}\right)\right)} w_{1}\left(e_{i j}\right) .
$$

The purpose of the implementation of the capability supported by $\Gamma_{i} v_{t}$ is to destroy the target process. Therefore, the value of the implementation of the capability is the value of the target. $Z\left(\Gamma_{i}\left(v_{t}\right)\right)$ is defined as:

$$
Z\left(\Gamma_{i}\left(v_{t}\right)\right)=f\left(v_{t}\right) .
$$

The cost of the implementation of the capability supported by $\Gamma_{i}\left(v_{t}\right)$ depends on the cost of the various relationships in $\Gamma_{i}\left(v_{t}\right)$. Based on the equation (1), $H\left(\Gamma_{i}\left(v_{t}\right)\right)$ is defined as:

$$
\begin{aligned}
& H\left(\Gamma_{i}\left(v_{t}\right)\right)=\sum_{e_{i j} \in E\left(\Gamma_{i}\left(v_{t}\right)\right)} w_{2}\left(e_{i j}\right), \\
& \Psi\left(\Gamma_{i}\left(v_{t}\right)\right)=\frac{Z\left(\Gamma_{i}\left(v_{t}\right)\right)}{I\left(\Gamma_{i}\left(v_{t}\right)\right) H\left(\Gamma_{i}\left(v_{t}\right)\right)} .
\end{aligned}
$$

For two paths $P_{m r}=e_{m k_{1}}, e_{k_{1} k_{2}}, \cdots, e_{k_{m} k_{n}}, e_{k_{n} r}$ and $P_{h l}=e_{h k_{1}^{\prime}}, e_{k_{1}^{\prime} k_{2}^{\prime}}, \cdots, e_{k_{m}^{\prime} k_{n}^{\prime}}, e_{k_{n}^{\prime} l}$, the joint product of the two paths is defined as $P_{m l}=P_{m r} \bullet P_{h l}$.

$$
P_{m l}=P_{m r} \bullet P_{h l}=\left\{\begin{array}{l}
\emptyset, \\
r \neq h \text { or } V\left(P_{m r}\right) \cap V\left(P_{h l}\right) \neq\left\{v_{h}, v_{l}\right\} \text { or } P_{m r}=\emptyset \text { or } P_{h l}=\emptyset, \\
e_{m k_{1}}, e_{k_{1} k_{2}}, \cdots, e_{k_{m} k_{n}}, e_{k_{n} r}, e_{h k_{1}^{\prime}}, e_{k_{1}^{\prime} k_{2}^{\prime}}, \cdots, e_{k_{m}^{\prime} k_{n}^{\prime}}, e_{k_{n}^{\prime} l}, \quad r=h .
\end{array}\right.
$$

The joint matrix of $G$ is defined as:

$$
A=\left(\alpha_{i j}\right)_{N \times N}= \begin{cases}e_{i j}, & \text { exits the relation from } v_{i} \text { to } v_{j} \\ \emptyset, & \text { else. }\end{cases}
$$

The path matrix is a matrix where the element in the matrix is the collection of paths in the combat network. That is, if $M=\left[m_{i j}\right]_{n \times k}$ is a path matrix, then $m_{i j}=\bar{P}_{i j}$. 
For two path matrices $M=\left[m_{i j}\right]_{n \times k}$ and $N=\left[n_{i j}\right]_{k \times q}$, the joint product of the two path matrices is defined as $Z=M \bullet N$ where $Z=\left[z_{i j}\right]_{n \times q}, z_{i j}=\bigcup_{r=1}^{k} m_{n r} \bullet n_{r q}(1 \leq i \leq n, 1 \leq j \leq$ $q)$.

Let $M^{k}=M^{k-1} \bullet A, k=2,3, \cdots, N$ with $M^{1}=A . M^{k}$ has the following properties:

1) The element $m_{i j}^{k}$ in $M^{k}$ is the collection of the directed paths from $v_{i}$ to $v_{j}$ and the length of each path is $k$.

2) $m_{i j}^{k}$ is the collection of the directed paths including the entity $v_{i}$ and the length of each path in the collection is $k$.

3) $m_{i j}^{k}$ is the path from $v_{i}$ to $v_{j}, j=1,2, \cdots, N$, and the length of the path is $k$.

4) $m_{i j}^{k}$ is the path from $v_{i}$ to $v_{j}, i=1,2, \cdots, N$, and the length of the path is $k$.

Based on the above properties, the algorithm to find the capability loops for target $v_{t}$ is given as following.

\section{Algorithm 1}

1) Let $M^{1}=A$ where $A$ is the joint matrix of $G$;

2) Let $k=2$ and $M^{k}=M^{k-1} \bullet A$;

3) If $m_{i j}^{k} \neq \emptyset, i=1,2, \cdots, N, j=1,2, \cdots, N$, then let $k=k+1$ and jump to Step 2), else jump to Step 4);

4) $m_{t t}^{k}$ is the collection of capability loops for target $v_{t}$ in $G$.

Based on the OODA loop, the process of attacking a target is the process of the observation, decision and action. One capability loop is a process of attacking the target including the stage of observation, decision and action. The more of the capability loops are in $\bar{\Gamma}\left(v_{t}\right)$, the more of the methods of attacking the target $v_{t}$ are, which decrease the uncertainty degree of the implementation of the capability aim to attacking the target $v_{t}$ and the overall decrease of the uncertainty degree is not the simple sum of the decrease supported by the single capability loop.

The evaluation index of the capability of the command network for target $v_{t}$ includes the uncertainty degree of the implementation of the capability denotes by $I\left(\bar{\Gamma}\left(v_{t}\right)\right)$, the value of the implementation of the capability denotes by $Z\left(\bar{\Gamma}\left(v_{t}\right)\right)$, the cost of the implementation of the capability denotes by $H\left(\bar{\Gamma}\left(v_{t}\right)\right)$ and the efficiency of the implementation of the capability denotes by $\Psi\left(\bar{\Gamma}\left(v_{t}\right)\right)$.

$$
I\left(\bar{\Gamma}\left(v_{t}\right)\right)=\frac{\prod_{i=1}^{m} I\left(\Gamma_{i}\left(v_{t}\right)\right)}{\sum_{i=1}^{m} I\left(\Gamma_{i}\left(v_{t}\right)\right)},
$$

where $m$ is the number of capability loops in $\bar{\Gamma}\left(v_{t}\right)$.

$$
\begin{aligned}
Z\left(\bar{\Gamma}\left(v_{t}\right)\right) & =f\left(v_{t}\right), \\
H\left(\bar{\Gamma}\left(v_{t}\right)\right) & =\sum_{i=1}^{m} H\left(\Gamma_{i}\left(v_{t}\right)\right), \\
\Psi\left(\bar{\Gamma}\left(v_{t}\right)\right) & =\frac{Z\left(\bar{\Gamma}\left(v_{t}\right)\right)}{I\left(\bar{\Gamma}\left(v_{t}\right)\right) H\left(\bar{\Gamma}\left(v_{t}\right)\right)} .
\end{aligned}
$$

$\bar{\Gamma}\left(v_{t}\right)$ describes all modes of attacking target by the combat network of CSoS. But the cost of each capability loop in $\bar{\Gamma}\left(v_{t}\right)$ is different. $\bar{\Gamma}_{C}\left(v_{t}, c\right)$ is the collection of capability loops which cost is not more than $c$ and named as $c$-cost cut of $\bar{\Gamma}\left(v_{t}\right) . \bar{\Gamma}_{I}\left(v_{t}, r\right)$ is the collection of capability 
loops which uncertainty degree is not less than $r$ and named as $r$-uncertainty degree cut of $\bar{\Gamma}\left(v_{t}\right)$.

$$
\begin{aligned}
& \bar{\Gamma}_{C}\left(v_{t}, c\right)=\left\{\Gamma_{i}\left(v_{t}\right) \mid \Gamma_{i}\left(v_{t}\right) \in \bar{\Gamma}\left(v_{t}\right) \text { and } H\left(\bar{\Gamma}\left(v_{t}\right)\right) \leq c\right\}, \\
& \bar{\Gamma}_{I}\left(v_{t}, r\right)=\left\{\Gamma_{i}\left(v_{t}\right) \mid \Gamma_{i}\left(v_{t}\right) \in \bar{\Gamma}\left(v_{t}\right) \text { and } H\left(\bar{\Gamma}\left(v_{t}\right)\right) \geq r\right\} .
\end{aligned}
$$

The role of the entity and the relation in the different capability loop is different. Core $V\left(v_{t}\right)$ is the core entities for $v_{t}$ and $\operatorname{Core} E\left(v_{t}\right)$ is the core relations for $v_{t}$.

$$
\begin{aligned}
& \operatorname{Core} V\left(v_{t}\right)=\left\{v \mid v \in V^{\prime}, v \in V \bigcap V\left(\Gamma_{i}\left(v_{t}\right)\right)\right\}, \\
& \operatorname{Core} E\left(v_{t}\right)=\left\{e \mid e \in V \bigcap E\left(\Gamma_{i}\left(v_{t}\right)\right)\right\} .
\end{aligned}
$$

The important of the entity in the command network can be measured by the change of the value of the evaluation index after removing the entity from the command network. The command network after removing the entity $v$ is denoted by $\bar{G} \cdot \bar{\Gamma}^{\prime}\left(v_{t}, v\right)$ is the collection of capability loops to attacking the target $v_{t}$ in $\bar{G}$. $\operatorname{IInf}\left(v, v_{t}\right)$ is the impact degree of entity $v$ to $G$ on the uncertainty degree for accomplishing the capability of attacking the target $v_{t}$. $H \operatorname{Inf}\left(v, v_{t}\right)$ is the impact degree of entity $v$ to $G$ on the cost for accomplishing the capability of attacking the target $v_{t}$. $\Psi \operatorname{Inf}\left(v, v_{t}\right)$ is the impact degree of entity $v$ to $G$ on the efficiency for accomplishing the capability of attacking the target $v_{t}$.

$$
\begin{aligned}
& \operatorname{Inf}\left(v, v_{t}\right)=\frac{\left|I\left(\bar{\Gamma}\left(v_{t}\right)\right)-I\left(\bar{\Gamma}^{\prime}\left(v_{t}, v\right)\right)\right|}{\max _{v_{i} \in V^{\prime}}\left|I\left(\bar{\Gamma}\left(v_{t}\right)\right)-I\left(\bar{\Gamma}^{\prime}\left(v_{t}, v_{i}\right)\right)\right|}, \\
& H \operatorname{Inf}\left(v, v_{t}\right)=\frac{\left|H\left(\bar{\Gamma}\left(v_{t}\right)\right)-H\left(\bar{\Gamma}^{\prime}\left(v_{t}, v\right)\right)\right|}{\max _{v_{i} \in V^{\prime}}\left|H\left(\bar{\Gamma}\left(v_{t}\right)\right)-H\left(\bar{\Gamma}^{\prime}\left(v_{t}, v_{i}\right)\right)\right|}, \\
& \Psi \operatorname{Inf}\left(v, v_{t}\right)=\frac{\left|\Psi\left(\bar{\Gamma}\left(v_{t}\right)\right)-\Psi\left(\bar{\Gamma}^{\prime}\left(v_{t}, v\right)\right)\right|}{\max _{v_{i} \in V^{\prime}}\left|\Psi\left(\bar{\Gamma}\left(v_{t}\right)\right)-\Psi\left(\bar{\Gamma}^{\prime}\left(v_{t}, v_{i}\right)\right)\right|} .
\end{aligned}
$$

The task of the command network is attacking all the targets instead of only one. The command network can be evaluated by the capability of attacking all targets. The evaluation index of the capability of command network includes the uncertainty degree of the implementation of the capability denotes by $I(G)$, the value of the implementation of the capability denotes by $Z(G)$, the cost of the implementation of the capability denotes by $H(G)$ and the efficiency of the implementation of the capability denotes by $\Psi(G)$.

$$
\begin{aligned}
I(G) & =\frac{\prod_{t=1}^{|T|} I\left(\bar{\Gamma}\left(v_{t}\right)\right)}{\sum_{t=1}^{|T|} I\left(\bar{\Gamma}\left(v_{t}\right)\right)}, \\
Z(G) & \left.=\sum_{t=1}^{|T|} f\left(v_{t}\right)\right), \\
H(G) & =\sum_{t=1}^{|T|} \sum_{i=1}^{m} H\left(\Gamma_{i}\left(v_{t}\right)\right), \\
\Psi(G) & =\frac{Z(G)}{I(G) H(G)} .
\end{aligned}
$$

$I(v, G)$ is the impact degree of entity $v$ to $G$ on the uncertainty degree for accomplishing the capability of attacking all targets. $H(v, G)$ is the impact degree of entity $v$ to $G$ on the cost 
for accomplishing the capability of attacking all targets. $\Psi(v, G)$ is the impact degree of entity $v$ to $G$ on the efficiency for accomplishing the capability of attacking all targets.

$$
\begin{aligned}
& I(v, G)=\sum_{v_{i} \in T} \frac{f\left(v_{i}\right)}{\sum_{v_{i} \in T} f\left(v_{i}\right)} \times \operatorname{Inf}\left(v, v_{i}\right), \\
& H(v, G)=\sum_{v_{i} \in T} \frac{f\left(v_{i}\right)}{\sum_{v_{i} \in T} f\left(v_{i}\right)} \times H \operatorname{Inf}\left(v, v_{i}\right), \\
& \Psi(v, G)=\sum_{v_{i} \in T} \frac{f\left(v_{i}\right)}{\sum_{v_{i} \in T} f\left(v_{i}\right)} \times \Psi \operatorname{Inf}\left(v, v_{i}\right),
\end{aligned}
$$

where $\frac{f\left(v_{i}\right)}{\sum_{v_{i} \in T} f\left(v_{i}\right)} \times \operatorname{HInf}\left(v, v_{i}\right)$ is the weight of different capability loop in $G$ which is decided by the value of the target in the capability loop.

\section{An Illustrative Example}

A combat network of CSoS is described in Figure 4. $T=\left\{V_{1}, V_{2}\right\}, V^{\prime}=\left\{V_{3}, V_{4}, V_{5}, V_{6}, V_{7}, V_{8}\right\}$, $S=\left\{V_{3}, V_{4}, V_{5}\right\}, D=\left\{V_{4}, V_{6}\right\}, I=\left\{V_{4}, V_{6}, V_{8}\right\}, f\left(V_{1}\right)=0.9, f\left(V_{2}\right)=0.8 . e_{44}$ : S-D is abbreviated to $e_{44}^{1}, e_{44}$ : D-I is abbreviated to $e_{44}^{2}$.

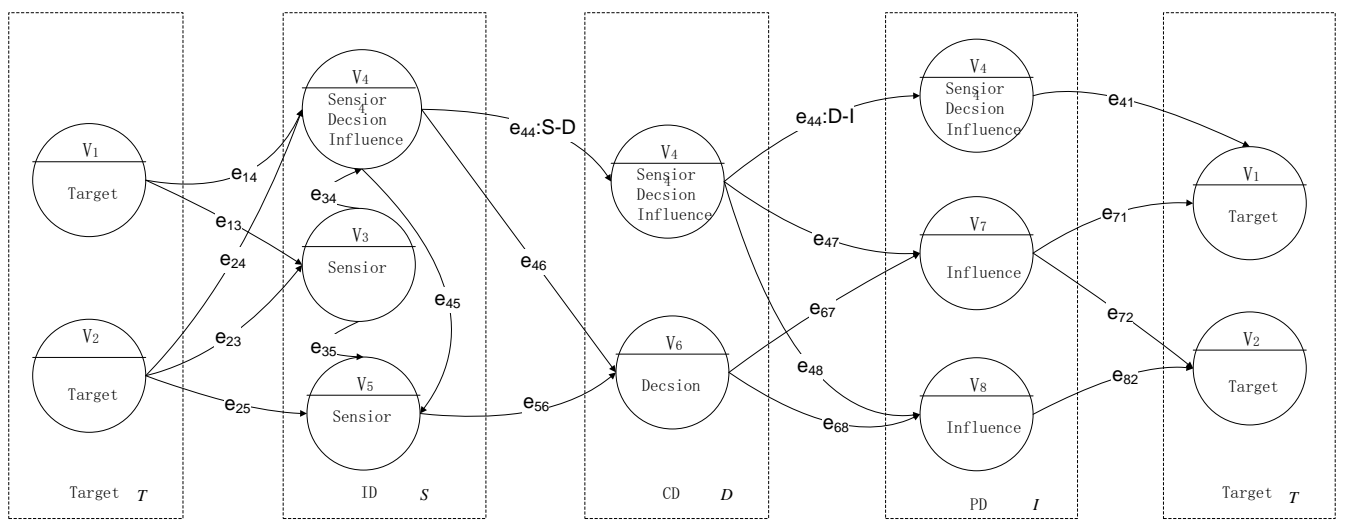

Figure 4 A combat network of CSoS

The implement degree and the cost of the implementation of the operational activity in the combat network are given in Table 1.

Table 1 Implement degree and cost of the implementation of the operational activity

\begin{tabular}{ccccccccccc}
\hline operational activity $e$ & $e_{13}$ & $e_{14}$ & $e_{23}$ & $e_{24}$ & $e_{25}$ & $e_{34}$ & $e_{35}$ & $e_{45}$ & $e_{44}^{1}$ & $e_{46}$ \\
\hline$w_{1}(e)$ & 0.177 & 0.123 & 0.128 & 0.125 & 0.105 & 0.105 & 0.051 & 0.083 & 0.001 & 0.03 \\
$w_{2}(e)$ & 0.3 & 0.4 & 0.4 & 0.3 & 0.3 & 0.3 & 0.2 & 0.5 & 0.05 & 0.6 \\
operational activity $e$ & $e_{56}$ & $e_{44}^{2}$ & $e_{47}$ & $e_{48}$ & $e_{67}$ & $e_{68}$ & $e_{41}$ & $e_{71}$ & $e_{72}$ & $e_{82}$ \\
$w_{1}(e)$ & 0.073 & 0.002 & 0.028 & 0.052 & 0.073 & 0.021 & 0.045 & 0.035 & 0.024 & 0.02 \\
$w_{2}(e)$ & 0.3 & 0.04 & 0.3 & 0.4 & 0.5 & 0.3 & 0.3 & 0.4 & 0.3 & 0.2 \\
\hline
\end{tabular}


Based on Algorithm 1, the capability loops in the combat network are $\bar{\Gamma}\left(v_{1}\right)=\left\{e_{14} e_{44}^{1} e_{44}^{2} e_{41}\right.$, $e_{14} e_{44}^{1} e_{47} e_{71}, e_{14} e_{46} e_{67} e_{71}, e_{13} e_{34} e_{44}^{1} e_{44}^{2} e_{41}, e_{13} e_{34} e_{44}^{1} e_{47} e_{71}, e_{13} e_{34} e_{46} e_{67} e_{71}, e_{13} e_{35} e_{56} e_{67} e_{71}$, $\left.e_{14} e_{45} e_{56} e_{67} e_{71}\right\}, \bar{\Gamma}\left(v_{2}\right)=\left\{e_{23} e_{35} e_{56} e_{68} e_{82}, e_{23} e_{34} e_{45} e_{56} e_{68} e_{82}, e_{24} e_{46} e_{68} e_{82}, e_{24} e_{46} e_{67} e_{72}\right.$, $\left.e_{24} e_{45} e_{56} e_{68} e_{82}, e_{25} e_{56} e_{68} e_{82}\right\}$.

The evaluation value of the capability loops for $v_{1}$ and $v_{2}$ are described in Figure 5 . The uncertainty degrees of the capability loops for $v_{1}$ (shown in Figure 5(a)) are not much different which is in $[0.171,0.42]$. But the costs of the capability loops (shown in Figure 5(a)) are very different which is in $[0.79,2.3]$. The capability loop with the max cost is $\Gamma_{3}\left(v_{1}\right)$ and its cost is 2.3. The capability loop with the min cost is $\Gamma_{1}\left(v_{1}\right)$ and its cost is 0.79 . There are differences between the efficiencies of the capability loops shown in Figure 5(a). The capability loop with the max efficiency is $\Gamma_{1}\left(v_{1}\right)$ and its efficiency is 6.66 . The capability loop with the min efficiency is $\Gamma_{6}\left(v_{1}\right)$ and its efficiency is 1.02 . The former is 6.5 times the latter. The capability loop for $v_{2}$ with the max efficiency is $\Gamma_{6}\left(v_{2}\right)$ shown in Figure 5(b). The capability loop with the min efficiency is $\Gamma_{2}\left(v_{2}\right)$ shown in Figure $5(\mathrm{~b})$. The former is 3.6 times the latter.

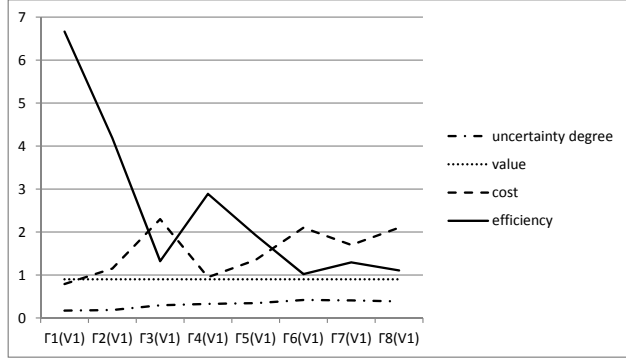

(a) $\bar{\Gamma}\left(v_{1}\right)$

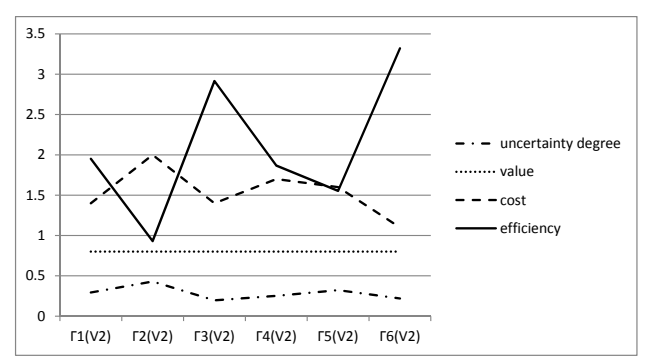

(b) $\bar{\Gamma}\left(v_{2}\right)$

Figure 5 The evaluation value of the capability loop for $v_{1}$ and $v_{2}$

It shows that there are different combat models which are denoted by the different capability loops can be chosen in the combat network of CSoS. These different combat models including different entities and cooperation between the entities can achieve the same combat task such as attacking the same target. But there are perhaps differences of the uncertainty degree, cost and efficiency between the different combat models. For example, if the task is attacking the target $v_{1}$, then the max efficiency can be achieved by $\Gamma_{1}\left(v_{1}\right)$ which includes the single entity $v_{4}$. If the task is attacking the target $v_{2}$, then the max efficiency can be achieved by $\Gamma_{6}\left(v_{2}\right)$ which is the cooperation among the entities $v_{2}, v_{5}, v_{6}, v_{8}$. If the task is attacking the target $v_{1}$ and $v_{2}$, then the max efficiency and the min cost can be achieved simultaneously by the cooperation among the entities $v_{2}, v_{4}, v_{5}, v_{6}, v_{8}$ and the other entities are unnecessary. The uncertainty degree of attacking $v_{2}$ is 0.29 and the uncertainty degree of attacking $v_{2}$ is 0.22 .

The evaluation value of the capability to attacking the target $v_{1}$ are $I\left(\bar{\Gamma}\left(v_{1}\right)\right)=0.000028$, $Z\left(\bar{\Gamma}\left(v_{1}\right)\right)=0.9, H\left(\bar{\Gamma}\left(v_{1}\right)\right)=12.44, \Psi\left(\bar{\Gamma}\left(v_{1}\right)\right)=2584$. The evaluation value of the capability to attacking the target $v_{2}$ are $I\left(\bar{\Gamma}\left(v_{2}\right)\right)=0.003522, Z\left(\bar{\Gamma}\left(v_{2}\right)\right)=0.8, H\left(\bar{\Gamma}\left(v_{2}\right)\right)=9.2, \Psi\left(\bar{\Gamma}\left(v_{2}\right)\right)=$ 24.7. It shows that the efficiency of the combat network to attacking $v_{1}$ is more than $v_{2}$.

The evaluation value of the capability to attack the target $v_{1}$ is changing with the changing of cost limit shown in Figure 6(a). The efficiency increases slowly before $c=1.35$ and increases 
fast after $c=1.35$. The uncertainty degree changes significantly with the changing of cost before $c=0.95$ but not after $c=1.15$. The impact of cost on uncertainty degree and efficiency is small where $1.15<c<1.35$. The evaluation value of the capability to attack the target $v_{2}$ are changing with the changing of cost limit shown in Figure 6(b). The impact of cost on uncertainty degree reaches maximum when $c=1.1$ and becomes smaller after $c=1.1$. The efficiency increases fast after $c=1.6$.

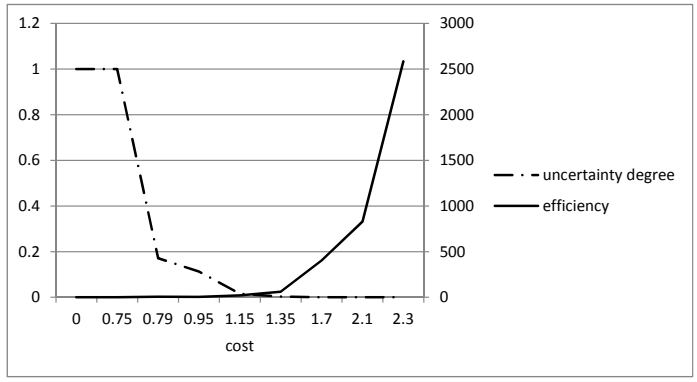

(a) $v_{1}$

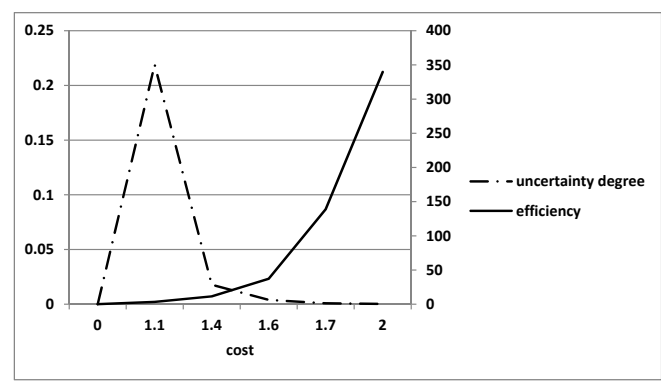

(b) $v_{2}$

Figure 6 The evaluation value change with the cost limit changing

The core entity is $\operatorname{Core} V\left(v_{1}\right)=\operatorname{Core} E\left(v_{1}\right)=\emptyset$ and the core relation is $\operatorname{Core} V\left(v_{2}\right)=\left\{v_{6}\right\}$, Core $E\left(v_{2}\right)=\emptyset$ in the command network, which means that the missing of $v_{6}$ will cause the missing of the capability of the command network to attack $v_{2}$.

The impact degree of the entity to the command network on the capability of attacking the target $v_{1}$ is shown in Figure 7(a). The missing of $v_{4}$ and $v_{7}$ have more impact than the missing of the others on the uncertainty degree for attacking the target $v_{1}$. The mission of $v_{8}$ has no impact on the uncertainty degree. The removal of $v_{3}, v_{5}, v_{6}, v_{8}$ can reduce more cost than the removal of the other entities and increase less uncertainty degree than the removal of the other entities.

The impact degree of the entity to the command network on the capability of attacking the target $v_{2}$ is shown in Figure 7(b). The missing of $v_{6}$ has more impact than the missing of the others on the uncertainty degree for attacking the target $v_{2}$. The missing of $v_{7}$ has more impact than the missing of the others on the efficiency for attacking the target $v_{2}$.

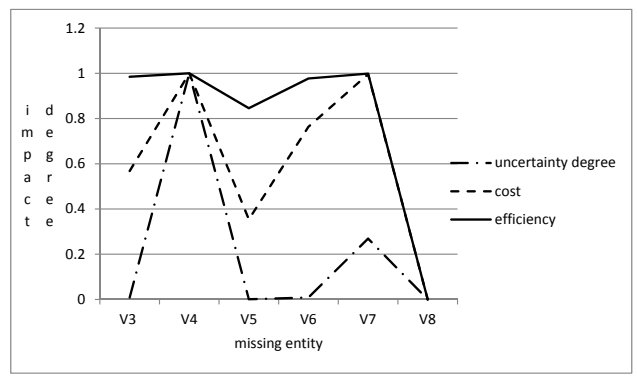

(a) $v_{1}$

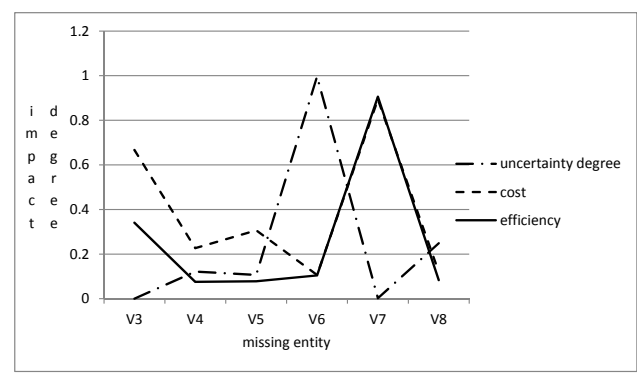

(b) $v_{2}$

Figure 7 The impact degree of the entity on the capability of attacking a target 
The evaluation value of the capability of the command network is $I(G)=0.0000278, Z(G)=$ 1.7, $H(G)=21.64, \Psi(G)=2825.8$. The impact degree of the entity on the capability of the command network for attacking all the targets is shown in Figure 8. The missing of $v_{4}$ and $v_{6}$ has more impact than the missing of the others on the uncertainty degree. The missing of $v_{7}$ has more impact than the missing of the others on the efficiency and cost. The missing of $v_{8}$ has little impact on the efficiency and the cost.

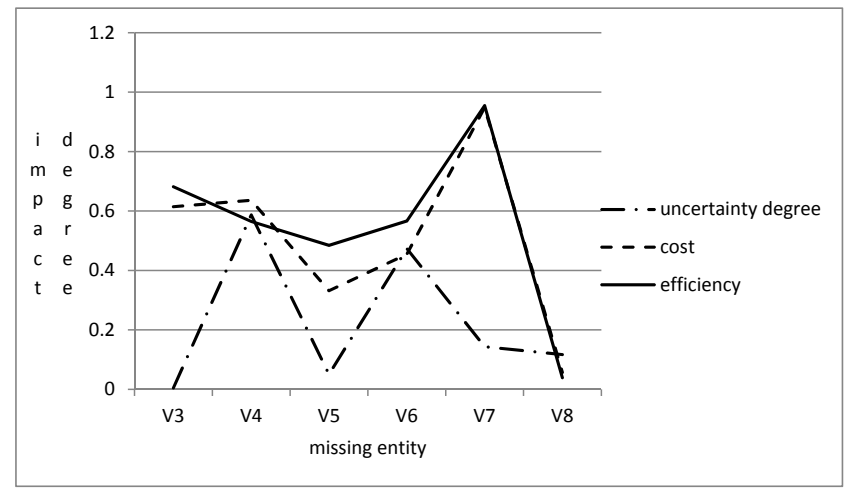

Figure 8 The impact degree of the entity on the capability of the command network

\section{Conclusion}

With the development of information technology, combat have increasingly reduced their reliance on weapon platforms and force concentration. Through combat network, the integration between various platforms is increasingly high while the distinction boundaries between different arms are becoming blurred. An information age CSoS model will require a transformation in military modeling philosophy and must therefore address these challenges by properly representing complex local behaviors, explicitly representing interdependencies and capturing the skewed distribution of networked performance. In addition, an information age CSoS model must capture both the attrition processes and the search and detection processes important to distributed, networked warfare. The implementation process of CSoS capability is analyzed. The process reveals the mechanism of CSoS capability implement which is the result of complex interactions between the entities in four domains through a sequence of action processes. The combat network model of CSoS is described which reflects the fundamental structure of networked combat. The capability loop is defined and the evaluation index of combat network of CSoS is proposed based on the capability loop analysis. Finally, an example is used to illustrate the methodology.

In the future, the combat model considering the CSoS conflicts will be studied which reflects more characteristics of the combat under the conditions of informationization.

\section{References}

[1] Alberts D S, Garstka J J, Stein F P, et al. Network-centric warfare: Developing and leveraging information superiority. Proceeding of CCRP, 2000.

[2] Kaplan J M. A new conceptual framework for enterprise-wide, net-centric system-of-systems engineering. Proceeding of International Conference on System of Systems Engineering, Los Angeles, CA, USA, 2006. 
[3] Deller S, Bell M I, Bowling S R, et al. Applying the information age combat model: Quantitative analysis of network centric operations. The International C2 Journal, 2009, 3(1): 1-25.

[4] Maier M W. Architecting principles for system of systems. Systems Engineering, 1998, 4(1): 267-284.

[5] Ricardo V, Baehren T, Boehm B, et al. A research agenda for systems of systems architecting, Incose International Symposium, 2007, 17(1): 1892-1908.

[6] Yang K W, Zhao Q S, Tan Y J, et al. System of systems requirements engineering techniques and methods. Beijing: Science Press, 2011.

[7] Zhao Q S, Yang K W, Chen Y W, et al. System of systems engineering and system of systems modeling. Beijing: National Defense Industry Press, 2013.

[8] Dou Y J, L L, Zhao Q S. Research on capability requirements generation of Weapon System-of-systems based on CRTAM model. Proceeding of 7th International Conference System of Systeme Engineering, July, Genoa, ITALY, 2012: 185-190.

[9] Kilicay N, Dagli C H. Methodologies for understanding behavior of system of systems. Proceeding of 7th International Conference System Engineering, April, 2007.

[10] Cares J R. An information age combat model. Technical Report, Alidade Incorporated, produced for the Director, Net Assessment, Office of the Secretary of Defense under Contract TPD-01-C-0023, 2004.

[11] Cares J. Distributed networked operations: The foundations of network centric warfare. Alidade Press, Newport, RI, 2005.

[12] Arquilla J, Karasik T. Chechnya: A Glimpse of Future Conflict? Studies in Conflict and Terrorism, 1999, 22(3): 207-229.

[13] Cebrowski A K, Garstka J J. Network-centric warfare: Its origin and future. Proceedings of the U.S. Naval Institute, 1998 124(1): 28-35.

[14] Li Z H, Liu S, Peng L B. Research on description models of SoS combat efficient based on complex network theory. Proceeding of International Conference on Computer Science and Network Technology, Harbin, China, 2011.

[15] Washburn A, Kress M. Combat modeling. International Series in Operations Research \& Management Science, 2009, 134: 133-160.

[16] Ancker C J, Gafarian A V. Modern combat models: A critique of their foundations. INFORMS, Catonsville, 1992.

[17] Engel J H. A verification of Lanchester's law. Journal of the Operations Research Society of America, 1954, 2(2): 163-171.

[18] Lepingwell J W R. The Laws of Combat?: Lanchester Reexamined. International Security, 1987, 12(1): 89-134.

[19] Bracken J. Lanchester models of the Ardennes campaign. Naval Research Logistics, 1995, 42(4): 559-577.

[20] Lucas T W, Turkes T. Fitting Lanchester models to the battles of Kursk and Ardennes. Naval Research Logistics, 2004, 51(1): 95-116.

[21] Koyuncu B, Bostanci E. Using Lanchester combat models to aid battlefield visualization. Proceeding of 2nd International Conference on Computer Science and Information Technology, Beijing, 2009.

[22] Schramm H C, Gaver D P. Lanchester for cyber: The mixed epidemic-combat model. Naval Research Logistics, 2013, 60(7): 599-605.

[23] Howard M, Karlin T S. An introduction to stochastic modeling. 4th Edition. Elsevier, 2010.

[24] Gilmer J B, Sullivan F J. Assignment of probabilities to events for combat simulation. Proceeding of Winter Simulation Conference, Austin, 2008.

[25] Khalid A, Bhatti A I, Hafiz R. A low complexity high resolution time-stepped stochastic ground combat simulation model. Proceeding of 7th International Multi Topic Conference, Islamabad, 2003.

[26] Protopopescu V, Santoro R T, Dockery J. Combat modeling with partial differential equations. European Journal of Operational Research, 1989, 38(2): 178-183.

[27] Poropudas J, Virtanen K. Game-theoretic validation and analysis of air combat simulation models. IEEE Transactions on Systems, Man \& Cybernetics — Part A: Systems \& Humans, 2010, 40(5): 1057-1070.

[28] Galster S M, Cummings M, Knott B, et al. Collaboration technologies for tactical command \& control: Performance, workload, and situation awareness. Proceeding of 2007 International Symposium on Collaborative Technologies and Systems, Orlando, Florida, USA, 2007.

[29] Liu J Y, Li X L. Application of colored petri net in command and control system. Proceeding of International 
Conference on Intelligent Human-Machine Systems and CyberneticsHangzhou, Zhejiang, China, 2009.

[30] Jiang N, Chen X, Hou C, et al. Warfare command decision making analysis of information support based on Lanchester equation. Proceeding of Chinese Control and Decision Conference, Xuzhou, China, 2010.

[31] Lee Y, Lee T. Network-based metric for measuring combat effectiveness. Defence Science Journal, 2014, 64(2): 115-122.

[32] Monsuur H. Assessing situation awareness in networks of cooperating entities: A mathematical approach. Military Operations Research, 2007, 12(3): 5-15.

[33] Rhee S H, Kim H S. Sohn S W. The effect of decentralized resource allocation in network-centric warfare. Proceeding of The International Conference on Information Networking. Bali, Indonesia, 2012.

[34] Butts C T. Revisiting the foundations of network analysis. Science, 2009, 325(5939): 414-416.

[35] Bohannon J. Investigating networks: The dark side. Science, 2009, 325: 410-411.

[36] Li H L, Cao Y Q, Jiang S Q. Research on fundamental properties of C2 system-oriented Complex Network. Proceeding of International Conference on Automatic Control and Artificial Intelligence, China, 2012.

[37] Qi Y B, Zhong L, Zhang L, et al. Distributed combat system of systems network modeling. Journal of Networks, 2013, 8(8): 1906-1912.

[38] Liu Y Y, Slotine J J, Barabsi A L. Controllability of complex networks. Nature, 2011, 473: 167-173.

[39] Qi Y B, Zhong L, Zhang L, et al. Joint combat system of systems modeling and effectiveness analysis based on complex networks. Advanced Materials Research, 2012, 591: 1589-1592.

[40] Grisogono A, Spaans M. Adaptive use of networks to generate an adaptive task force. Proceeding of 13th International Command and Control Research and Technology Symposium, CCRP Press, 2008.

[41] Rosseau R, Breton R. The M-OODA: A model incorporating control functions and teamwork in the OODA loop. Proceeding of Comma and control research and technology symposium. San Diego, Calif, 2004.

[42] Brehmer B. The dynamic OODA loop: Amalgamating boyd's OODA loop and the cybernetic approach to command and control. Proceeding of 10th International Command \& Control Research \& Technology Symposium, Herl'any, 2005.

[43] United States Department of Defense. Integrated Air and Missile Defense Joint Integrating Concept Version 2.3. September 17, 2004. 